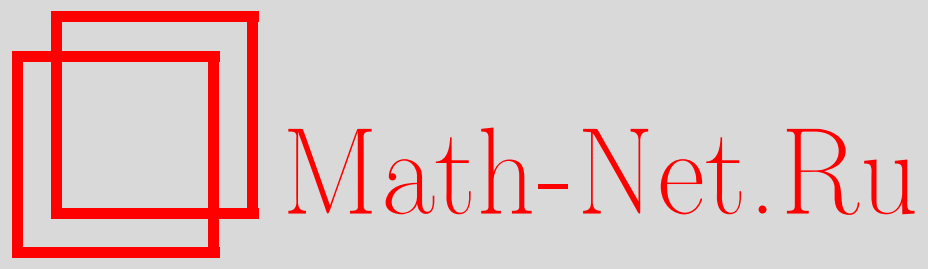

Ю. Г. Кудряшов, Костистые аттракторы, Функи. анализ и его прил., 2010, том 44, выпуск 3, 73-76

DOI: https://doi.org/10.4213/faa2997

Использование Общероссийского математического портала MathNet.Ru подразумевает, что вы прочитали и согласны с пользовательским соглашением

http://www . mathnet.ru/rus/agreement

Параметры загрузки:

IP : 35.173 .219 .149

26 апреля 2023 г., 15:21:56

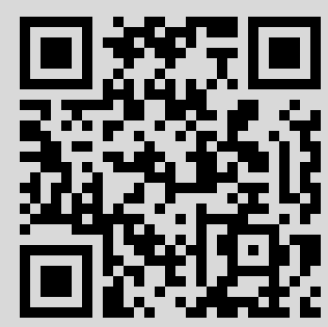




\title{
Костистые аттракторы*
}

\author{
(c) 2010. Ю. Г. Кудряшов
}

1. Введение. Вопросы о возможных структурах аттракторов являются одними из важнейших в теории динамических систем.

В простейших случаях аттракторы - это многообразия. Широко известны типичные динамические системы (аттрактор Лоренца, соленоид СмейлаВильямса), аттракторы которых локально устроены как прямое произведение многообразия на канторово множество. В этой заметке построен типичный (пока только в классе ступенчатых косых произведений) пример системы, аттрактор которой устроен по-другому, - является «костистым».

Напомним, что косым произведением называется динамическая система вида $F: Y \times Z \rightarrow Y \times Z,(y, z) \mapsto(f(y), h(y, z))$.

Будем называть аттрактор $A$ косого произведения $F: Y \times Z \rightarrow Y \times Z$ косmистым, если он является объединением графика непрерывной функции $g: D \rightarrow Z$, определенной на некотором подмножестве $D \subset Y$, и несчетного числа вертикальных отрезков («костей»), лежащих в замыкании этого графика.

Через $\Sigma^{k}$ обозначим пространство двусторонних последовательностей из чисел от 0 до $k-1$ с мерой Бернулли $\mu$, соответствующей некоторому распределению вероятностей $p_{0}, \ldots, p_{k-1}$, и «k-адической» метрикой

$$
d(\omega, \widetilde{\omega})=k^{-n(\omega, \widetilde{\omega})},
$$

где $n(\omega, \widetilde{\omega})-$ наименьшее число $n$, для которого $\omega_{i} \neq \widetilde{\omega}_{i}$ или $\omega_{-i} \neq \widetilde{\omega}_{-i}$. Пусть $\sigma: \Sigma^{k} \rightarrow \Sigma^{k},(\sigma \omega)_{j}=\omega_{j+1},-$ сдвиг Бернулли.

Рассмотрим пространство $C^{1}$-гладких ступенчатых косых произведений над сдвигом Бернулли со слоем $I=[0 ; 1]$, т. е. динамических систем вида

$$
F: \Sigma^{k} \times I \rightarrow \Sigma^{k} \times I, \quad(\omega, x) \mapsto\left(\sigma \omega, f_{\omega_{0}}(x)\right),
$$

где $f_{0}, \ldots, f_{k-1}: I \rightarrow I$ - непрерывно дифференцируемые функции. Это пространство естественно снабдить топологией $k$-й степени пространства $C^{1}[0 ; 1]$.

Основным результатом данной статьи является следующая теорема:

Теорема 1. Для любого $k \geqslant 2$ существует открытое непустое подмножество пространства $C^{1}$-гладких ступенчатых косых произведений над сдвигом Бернулли $\sigma: \Sigma^{k} \rightarrow \Sigma^{k}$, такое, что для любого его элемента $F$ выполнены следуюшие условия:

(1) максимальный аттрактор $A_{\max }=\bigcap_{n \geqslant 0} F^{n}\left(\Sigma^{k} \times I\right)$ является обгединением графика $\Gamma$ функиии $g: D \rightarrow I$, непрерывной на $D$, и множества вертикальных отрезков («костей»), по одному в каждом слое $\{\omega\} \times I, \omega \notin D$;

(2) $\operatorname{dim}_{H}(\Omega)<\operatorname{dim}_{H}\left(\Sigma^{k}\right)$, где $\Omega=\Sigma^{k} \backslash D-$ множество слоев, содержащих кости; кроме того, $\mu(\Omega)=0$;

(3) множество $\Omega$ континуально и всюду плотно в $\Sigma^{k}$;

* Работа выполнена при поддержке грантов РФФИ 07-01-00017-а, РФФИ 10-01-00739-а и РФФИ-СNRS 05-01-02801-НЦНИЛ_а. 
(4) для любого подмножества $S \subset \Sigma^{k}$ полной меры максималъный аттрактор $A_{\max }(F)$ совпадает с замыканием пересечения $A_{\max }(F) \bigcap(S \times I) ;$ в частности, «кости» лежат в замыкании графика $\Gamma$;

(5) максимальный аттрактор отображения $F$ совпадает с его милноровским аттрактором: $A_{\max }(F)=A_{M}(F)$.

Замечание 2. Максимальный аттрактор отображения $F$ обязан содержаться в множестве $\Sigma^{k} \times J$, где $J$ - выпуклая оболочка множества неподвижных точек послойных отображений $f_{i}$.

Ключевую роль в доказательстве теоремы 1 играет следующее достаточное условие.

Теорема 3. Пусть строго монотонные отображения $f_{0}, \ldots, f_{k-1}: I \rightarrow I$ обладают следующими свойствами:

(1) существует конечный набор композищий конечной длины из отображсений $f_{i}$, такой, что пересечение образов отрезка I под действием этих композииий пусто;

(2) существует композиция конечной длины из отображсний $f_{i}$, одна из неподвижных точек которой - репеллер;

(3) существует конечный набор конечных композищий отображсений $f_{i}$, такой, что каждая композиция из этого набора является сжатием на I, а образы отрезка $J=J\left(f_{0}, \ldots, f_{k-1}\right)$ при этих композициях покрывают отрезок $J$.

Тогда для соответствующего ступенчатого косого произведения $F: \Sigma^{k} \times$ $I \rightarrow \Sigma^{k} \times I$ выполнены заключения теоремы 1 .

Перед тем как доказывать эту теорему, приведем пример открытого множества ступенчатых косых произведений, для которых ее условия выполнены. Тем самым мы выведем теорему 1 из теоремы 3.

При $k \geqslant 3$ возьмем отображения $f_{0}$ и $f_{1}$, сжимающие к двум разным точкам $x_{0}<x_{1}$ отрезка $I$, причем $0,75<f_{0}^{\prime}, f_{1}^{\prime}<1$. Тогда для достаточно большого $n$ отрезки $f_{0}^{n}(I)$ и $f_{1}^{n}(I)$ не пересекаются, т. е. выполнено условие $(1)$. Выберем отображения $f_{2}, \ldots, f_{k-1}$ так, что $f_{i}(I) \subset\left(x_{0}, x_{1}\right)$, причем хотя бы у одного из этих отображений есть репеллер. Это гарантирует выполнение условия (2). Кроме того, это означает, что $J=\left[x_{0}, x_{1}\right]$, и из неравенства $0,75<f_{0}^{\prime}, f_{1}^{\prime}$ следует условие (3). Ясно, что описанный в этом абзаце класс наборов отображений непуст и открыт в $C^{1}$-топологии.

Пример для $k=2$ построить немного сложнее. Пусть $f_{0}-$ кусочно-линейное отображение с вершинами $(0,0),(0,6,0,2),(1,0,8)$, а $f_{1}$ - кусочно-линейное отображение с вершинами $(0,0,15),(0,4,0,8),(1,1)$. Несложно проверить, что образы композиций $f_{0}^{5}$ и $f_{1}^{5}$ не пересекаются, а у композиции $f_{0} f_{1}$ есть отталкивающая неподвижная точка. Заметим, что композиции $f_{0}^{3}, f_{0}^{2} f_{1}, f_{1}^{2} f_{0}$ и $f_{1}^{5}$ удовлетворяют последнему условию теоремы 3. Осталось немного сгладить изломы и взять окрестность полученной системы в топологии $C^{1}$.

В следующих разделах мы поочередно выведем заключения теоремы 1 из условий теоремы 3.

2. Структура аттрактора. Заметим, что со слоем над последовательностью $\omega$ максимальный аттрактор пересекается по множеству $I_{\omega}=\bigcap_{n=1}^{\infty} I_{\omega, n}$, где $I_{\omega, n}=f_{\omega_{-1}} \circ \cdots \circ f_{\omega_{-n}}(I)$. Поскольку отрезки $I_{\omega, n}$ вложены друг в друга, $I_{\omega}$ - точка или отрезок. Таким образом, максимальный аттрактор является объединением графика некоторой функции $g$ и множества вертикальных отрезков. 
Докажем теперь непрерывность функции $g$. Пусть $\left(\omega^{(0)}, x^{(0)}\right)$ - точка графика $\Gamma, \varepsilon>0$. Тогда для достаточно большого $n$ отрезок $I_{\omega(0), n}$ содержится в интервале $\left(x^{(0)}-\varepsilon, x^{(0)}+\varepsilon\right)$. Значит, $I_{\omega, n} \subset\left(x^{(0)}-\varepsilon, x^{(0)}+\varepsilon\right)$ для любой последовательности $\omega$, совпадающей с $\omega^{(0)}$ на $[-n ;-1]$, т. е. функция $g$ непрерывна на области определения.

3. Хаусдорфова размерность и мера. Докажем сначала следующую леммy.

Лемма 4. Пусть отображения $f_{0}, \ldots, f_{k-1}: I \rightarrow I$ удовлетворяют условию (1) теоремы 3. Тогда для любой точки $x \in I$ хаусдорфова размерность множества $\Omega^{x}$ последовательностей $\omega$, для которых $x \in I_{\omega}$, менъше $\operatorname{dim}_{H} \Sigma^{k}$.

Доказательство. Пусть $N$ - наибольшая из длин композиций из условия (1) теоремы 3 и $\Omega_{l}^{x}=\left\{w \in\{0, \ldots, k-1\}^{N l} \mid x \in f_{w}(I)\right\}$. Тогда левая часть любой последовательности из $\Omega^{x}$ начинается с последовательности из $\Omega_{l}^{x}$, а значит, множество $\Omega^{x}$ содержится в объединении $k^{N l+1}\left|\Omega_{l}^{x}\right|$ шаров радиуса $k^{-N l-1}$. Оценим $\left|\Omega_{l}^{x}\right|$.

Любая последовательность $w \in \Omega_{l+1}^{x}$ имеет вид $u v,|u|=N, v \in \Omega_{l}^{x}$, а значит, $\left|\Omega_{l+1}^{x}\right| \leqslant k^{N}\left|\Omega_{l}^{x}\right|$. Более того, для каждой последовательности $\widetilde{v} \in \Omega_{l}^{x}$ найдется последовательность $\widetilde{u}$ длины $N$, такая, что $f_{\widetilde{v}}^{-1}(x) \notin f_{\widetilde{u}}(I)$, т. е. $\widetilde{w}=\widetilde{u} \widetilde{v} \notin \Omega_{l+1}^{x}$. Следовательно, $\left|\Omega_{l+1}^{x}\right| \leqslant\left(k^{N}-1\right)\left|\Omega_{l}^{x}\right|$ и $\left|\Omega_{x}^{l}\right| \leqslant\left(k^{N}-1\right)^{l}$.

Таким образом, множество $\Omega^{x}$ лежит в объединении не более чем $k\left(k^{2 N}-k^{N}\right)^{l}$ шаров радиуса $k^{-N l-1}$; следовательно, хаусдорфова размерность множества $\Omega^{x}$ не превосходит $\log _{k^{N}}\left(k^{2 N}-k^{N}\right)<2=\operatorname{dim}_{H} \Sigma^{k}$.

Поскольку $\Omega$ содержится в счетном объединении $\bigcup_{q=1}^{\infty} \bigcup_{p=0}^{q} \Omega^{p / q}$, а хаусдорфова размерность каждого из этих множеств не превосходит $\log _{k^{N}}\left(k^{2 N}-k^{N}\right)$, хаусдорфова размерность множества $\Omega$ также не превосходит этого числа.

Аналогично лемме 4 доказывается тот факт, что мера любого из множеств $\Omega^{x}$ равна нулю. Следовательно, мера множества $\Omega$ также равна нулю, и второе утверждение теоремы доказано.

4. Континуальность и всюду плотность. В силу условия (2) теоремы 3 существует композиция $f_{\alpha}=f_{\alpha_{1}} \circ \cdots \circ f_{\alpha_{|\alpha|}}$, одна из неподвижных точек которой - репеллер. Пусть $\tilde{I} \subset I$ - окрестность репеллера, такая, что $f_{\alpha}(\tilde{I}) \supset \tilde{I}$. Тогда для любой последовательности вида $\omega=\ldots \alpha \alpha \ldots \alpha \omega_{-n} \ldots \omega_{-1} \omega_{0} \ldots$ отрезок $I_{\omega}$ содержит, как минимум, отрезок $f_{\omega_{-1}} \circ \cdots \circ f_{\omega_{-n}}(\tilde{I})$. Осталось заметить, что множество таких последовательностей континуально и всюду плотно.

5. Замыкание аттрактора над множеством полной меры. Пусть $(\omega, x)$ - точка максимального аттрактора. Обозначим через $U_{N}(\omega)$ множество последовательностей, совпадающих с $\omega$ на отрезке $[-N, N]$, а через $V_{\varepsilon}(x) \varepsilon$-окрестность точки $x$. Для доказательства четвертого утверждения теоремы достаточно построить точку, принадлежащую пересечению $\left(U_{N}(\omega) \times V_{\varepsilon}(x)\right) \cap(S \times I) \cap$ $A_{\max }$.

Применим композицию $h=f_{\omega_{-n}}^{-1} \circ \cdots \circ f_{\omega_{-1}}^{-1}$. Заметим, что точка $\left(\omega^{\prime}, x^{\prime}\right)=$ $\left(\sigma^{-n} \omega, h(x)\right)$ принадлежит $A_{\max }$, откуда следует, что $x^{\prime} \in J$. Применяя достаточное количество раз условие (3), мы можем найти сколь угодно сильно сжимающую композицию отображений $f_{i}$, образ которой содержит точку $x^{\prime}$ (см. [2]). В частности, можно найти последовательность $w$, такую, что $f_{w}(I) \subset$ 
$h\left(V_{\varepsilon}(x)\right)$. Выберем последовательность $\widetilde{\omega} \in S$, левый «хвост» которой имеет вид $\ldots w \omega_{-n} \ldots \omega_{-1}$, и точку $\widetilde{x} \in I_{\widetilde{\omega}}$. Тогда $I_{\widetilde{\omega}} \subset f_{w \omega_{-n} \ldots \omega_{-1}}(I) \subset V_{\varepsilon}(x)$; следовательно, пара $(\widetilde{\omega}, \widetilde{x})$ принадлежит пересечению $\left(U_{N}(\omega) \times V_{\varepsilon}(x)\right) \cap(S \times I) \cap A_{\max }$, и утверждение (4) выполнено.

6. Совпадение аттракторов. Заметим сначала, что точка $\left(\omega^{\prime}, x^{\prime}\right)$ графика $\Gamma$ принадлежит $\omega$-предельному множеству точки $(\omega, x)$, если последовательность $\omega^{\prime}$ принадлежит $\omega$-предельному множеству последовательности $\omega$. Действительно, если последовательности $\sigma^{n} \omega$ и $\omega^{\prime}$ совпадают на отрезке $[-N, N]$, то $f_{\sigma^{n} \omega}(x) \in I_{\omega^{\prime}, N}$, а отрезки $I_{\omega^{\prime}, N}$ пересекаются только по точке $x^{\prime}$.

Поскольку милноровский аттрактор сдвига Бернулли совпадает со всем множеством $\Sigma^{k}$, милноровский аттрактор рассматриваемого косого произведения содержит все точки графика. Осталось воспользоваться предыдущим утверждением теоремы.

7. Обобщения. Следующая теорема является непосредственным обобщением леммы 4.

Теорема 5. Пусть $M$ - сепарабельное топологическое пространство, $f_{1}, \ldots, f_{k}: M \rightarrow M u F-$ соответствующее ступенчатое косое произведение на $\Sigma^{k} \times M$. Пусть существуют две композиции конечной длины из $f_{i}$, образы которых не пересекаются. Обозначим через $\Omega$ множество последовательностей $\omega \in \Sigma^{k}$, для которых пересечение $A_{\max }(F) \cap(\{\omega\} \times M)$ имеет непустую внутренность в слое $\{\omega\} \times M$. Тогда $\operatorname{dim}_{H} \Omega<\operatorname{dim}_{H} \Sigma^{k}$.

8. Благодарности. Автор благодарен Ю. С. Ильяшенко за постановку задачи и полезные обсуждения. Автор также благодарен рецензенту за совет переформулировать основную теорему и В. А. Клепцыну за замечания к тексту статьи.

\title{
ЛитерАТУРА
}

[1] J. Milnor, Comm. Math. Phys., 99, 177-195. [2] J. Hatchinson, Indiana Univ. Math. J., 30:5 (1981), 713-747.

Московский государственный университет Независимый московский университет

Ecolé Normale Supériore de Lyon

e-mails: urkud.urkud@gmail.com,urkud@mccme.ru

Поступило в редакцию 13 июля 2009 г.

\section{УДК 517.51}

\section{Об оптимальном гармоническом синтезе по неточно заданному спектру*}

\author{
(C) 2010. Г. Г. МАГАРИЛ-ИЛЬЯЕВ, К. Ю. ОСИПЕНКО
}

Работа посвящена построению семейства оптимальных методов восстановления производных функций по неточно заданному на конечном отрезке преобразованию Фурье этих функций. Точная постановка задачи такова. Пусть $n \in \mathbb{N}$

*Работа выполнена при финансовой поддержке Российского фонда фундаментальных исследований (гранты № 08-01-00450 и №09-01-90360). 